\title{
Head-on collision of two solitary waves and residual falling jet formation
}

\author{
J. Chambarel, C. Kharif, and J. Touboul \\ Institut de Recherche sur les Phénomènes Hors Equilibre, Marseille, France
}

Received: 30 October 2008 - Revised: 30 January 2009 - Accepted: 30 January 2009 - Published: 17 February 2009

\begin{abstract}
The head-on collision of two equal and two unequal steep solitary waves is investigated numerically. The former case is equivalent to the reflection of one solitary wave by a vertical wall when viscosity is neglected. We have performed a series of numerical simulations based on a Boundary Integral Equation Method (BIEM) on finite depth to investigate during the collision the maximum runup, phase shift, wall residence time and acceleration field for arbitrary values of the non-linearity parameter $a / h$, where $a$ is the amplitude of initial solitary waves and $h$ the constant water depth. The initial solitary waves are calculated numerically from the fully nonlinear equations. The present work extends previous results on the runup and wall residence time calculation to the collision of very steep counter propagating solitary waves. Furthermore, a new phenomenon corresponding to the occurrence of a residual jet is found for wave amplitudes larger than a threshold value.
\end{abstract}

\section{Introduction}

In this paper we investigate the head-on collision of two equal and two unequal solitary waves which are computed by using the algorithm developed by Tanaka (1986). The symmetric case is equivalent, in the absence of viscosity, to the reflection of one solitary wave by a vertical wall. A lot of works considered analytically, numerically and experimentally this problem, calculating namely the maximum run-up amplitude, phase shift due to the collision and wall residence time. An exhaustive review of this problem is given below. In order to check the validity of their numerical method Chan and Street (1970) considered the run-up of a solitary wave on a vertical wall and compared their results with available

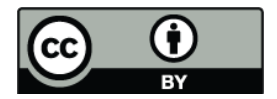

Correspondence to: J. Chambarel (chambarel@irphe.univ-mrs.fr) experimental data. Byatt-Smith (1971) calculated analytically to second-order approximation the interaction of two solitary waves going in opposite directions. Later on, ByattSmith (1988) investigated the head-on collision of two equal solitary waves or the reflection of a solitary wave by a vertical wall. Using a perturbation expansion of the Euler equation, he derived interaction equations and showed analytically that the amplitude of the solitary wave after reflection is reduced. The change is shown to be of fifth-order in wave amplitude. This loss of amplitude is due to the presence of the third-order dispersive tail. Oikawa and Yajima (1973) used a singular perturbation method developed to secondorder to study the interaction between two solitary waves which propagate in opposite directions. They provided an estimate of the phase shifts in the collision process of the two solitary waves. Maxworthy (1976) conducted experiments on the head collision of two solitary waves. He found that some of the theories are in qualitative but not quantitative agreement with his experimental results. Namely, he claimed that the phase shift is independent of initial amplitude in contrast with available theoretical results. Within the framework of the Lagrangian formulation of the equations, Temperville (1979) investigated the reflection of a solitary wave on a rigid wall. Namely, he derived the leading-order asymptotic formula for the phase shift between the incident wave and the reflected wave and found it was difficult to conclude, as Maxworthy did, that there is a constant phase shift. This disagreement is partially explained by Fenton and Rienecker (1982) who emphasized the sensitivity of the results to the measurement locations. Within the framework of the shallow water wave equations, Pelinovsky et al. (1999) derived an analytic expression of the maximum run-up amplitude of tsunami waves. Su and Mirie (1980) found analytically that the wave emerging from the collision of two solitary waves preserve their original identities to the third order of accuracy. Furthermore, the collision generates secondary wave groups (the dispersive tail) trailing behind their primary 
solitary waves. They calculated the maximum run-up amplitude of the two colliding waves up to third-order. In a numerical study based on the approximate equations derived by Su and Gardner (1969), Mirie and Su (1982) checked the phase shifts and maximum amplitude of a collision with a corresponding perturbation calculation and compared with experiments. They found a wave train trailing behind each of emerging solitary waves from the head-on collision. The properties of the wave train were in agreement with those of the perturbation solution. After the collision, the solitary waves recover almost all of their original amplitude for the length of time in the numerical simulation. They showed the difference persists ( $2 \%$ of their original value) and accounts for the energy residing in the wave train. Fenton and Rienecker (1982) developed a numerical method based on a Fourier decomposition for solving nonlinear water wave problems, namely solitary wave interactions. They investigated the maximum run-up at the wall and the phase shift during the interaction. The first to report on the wall residence time which is the time the wave crest remains attached to the wall, was Temperville (1979). Later on, Power and Chwang (1984) confirmed his results through an Eulerian approach. They considered the reflection of a solitary wave by solving the Boussinesq equations analytically as well as numerically. Cooker et al. (1997) using a Boundary Integral Equation Method (BIEM) for solving the fully nonlinear equations, showed that the wall residence time provides an unambiguous characterization of the phase shift incurred during the reflection. Bona and Chen (1998) as did Power and Chwang (1984) considered a Boussinesq system to study the head-on collision of solitary waves. More recently, Craig et al. (2006) considered the fully nonlinear equations to study numerically the run-up, phase lag, and generation of a residual from the head-on collision of two solitary waves. In addition to the symmetric case, they investigated the asymmetric case corresponding to two counter propagating solitary waves of different amplitudes. Note that Byatt-Smith (1971), Su and Mirie (1980), Mirie and Su (1982) and Bona and Chen (1998) considered the asymmetric case too.

Among the fully nonlinear water wave equations, Cooker et al. (1997) and Chan and Street (1970) considered waves with normalized amplitude $a / h$ up to 0.70 . The latter authors were only concerned with the maximum run-up whereas Cooker and co-authors investigated more deeply the interaction. Nevertheless, none of them discussed about a new phenomenon peculiar to head-on collision of very steep solitary waves: the formation of a residual thin jet. This jet is observed for the first time during the collision of the two counter propagating solitary waves

In Sect. 2 we present the mathematical statement of the water wave problem, and we describe briefly the numerical method. Section 3 is devoted to the maximum runup, phase shift, wall residence time and acceleration field for arbitrary amplitude of the two incident solitons, up to $a / h=0.80$. In this section, we discussed the generation of a residual jet when the initial amplitude of the counter propagating solitary waves is very large. The second part of the Sect. 3 reports on the collision of two solitary waves of different amplitudes. During the formation of the residual jet, the curvature becomes important and surface tension effect may be taken into account.

\section{Mathematical formulation and numerical method}

\subsection{Basic equations}

The problem is solved by assuming that the fluid is inviscid, incompressible, and the motion irrotational. Hence the velocity field is given by $\boldsymbol{u}=\nabla \phi$ where the velocity potential $\phi(x, z, t)$ satisfies the Laplace's equation which is solved in a domain bounded by the free surface, a horizontal solid bottom and two vertical solid walls located at the ends of the numerical domain. The horizontal and vertical coordinates are $x$ and $z$ respectively whereas $t$ is time. The still-water level lies at $z=0$, and the horizontal impermeable bed lies at $z=-h$. The dynamic free surface condition states that the pressure at the surface, $z=\eta(x, t)$, is equal to 0 . Assuming the free surface to be impermeable, the problem to be solved is the Laplace equation with the kinematic, dynamic and bottom conditions.

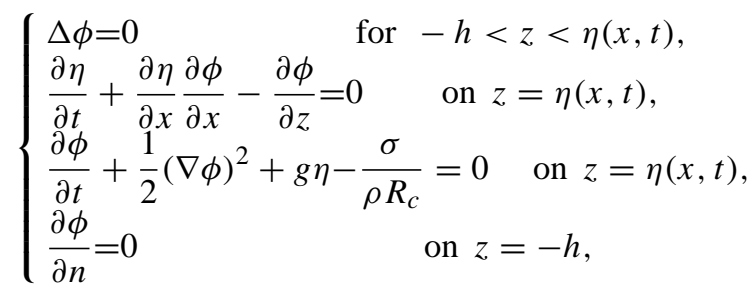

where $g$ is the acceleration due to gravity, $\rho_{\omega}$ is the water density, $\sigma$ is the surface tension coefficient and $R_{c}$ is the radius of curvature. During the formation of the residual jet, the curvature becomes important and surface tension effect may be taken into account.

\subsection{Numerical method}

A Boundary Integral Equation Method (BIEM) is used to solve the system of Eq. (1) with a mixed Euler Lagrange (MEL) time marching scheme. For more details about this numerical method see the papers by Touboul et al. (2006) and Touboul and Kharif (2009).

The Green's second identity is used to solve Laplace's equation for the velocity potential

$$
\begin{aligned}
c(Q) \phi(Q)= & \int_{\partial \Omega} \phi(P) \frac{\partial G}{\partial n}(P, Q) d l \\
& -\int_{\partial \Omega} \frac{\partial \phi}{\partial n}(P) G(P, Q) d l,
\end{aligned}
$$




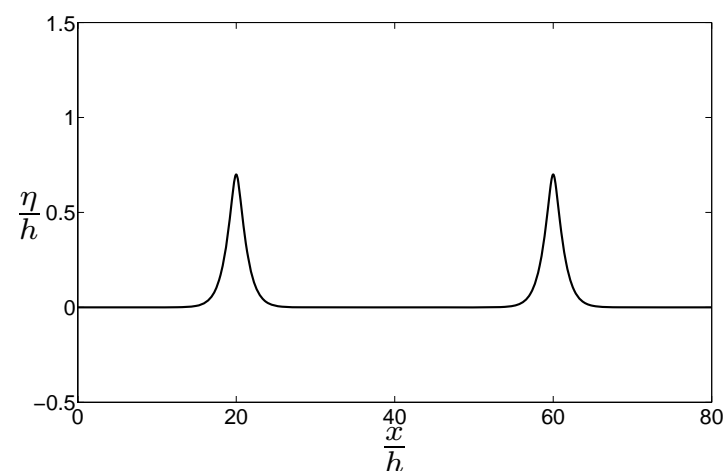

Fig. 1. Initial condition : Free surface elevation as a function of abscissa.

where $G$ is the free space Green's function. The fluid domain boundary $\partial \Omega$ is $\partial \Omega_{F} \cup \partial \Omega_{B}$, the union of the free surface $\partial \Omega_{F}$ and solid boundaries $\partial \Omega_{B}$. The unit normal vector $\boldsymbol{n}$ points outside the fluid domain. The unknowns are $\partial \phi / \partial n$ on $\partial \Omega_{F}$ and $\phi$ on $\partial \Omega_{B}$. Let $P$ and $Q$ denote two points of the domain and $c(Q)$ is the angle between two consecutive panels defined as follows

$c(Q)=\left\{\begin{array}{l}0 \quad \text { if } Q \text { is outside the fluid domain, } \\ \alpha \quad \text { if } Q \text { is on the boundary, } \\ -2 \pi \quad \text { if } Q \text { is inside the fluid domain, }\end{array}\right.$

where $\alpha$ is the inner angle relative to the fluid domain at point Q along the boundary.

Time stepping is performed using a fourth order Runge \& Kutta scheme, with a constant time step.

The velocity and acceleration fields are calculated using a finite-difference method.

\subsection{Initial conditions}

We consider a rectangular wave tank of length $L$ and constant depth $h$ with two vertical solid walls located at its ends. The horizontal length of the domain, $L$, is assumed to be large enough to avoid any perturbation generated from the vertical walls during the computational time of the simulations on the solitary wave collision occurring in the middle of the tank. We impose the impermeability condition on the vertical walls and horizontal bottom. Hence, the boundary conditions are $\partial \phi / \partial n=0$ on the walls and bottom. The initial free surface is represented by two solitary waves computed without approximation with the method of Tanaka. The free surface elevation is shown in Fig. 1 for two waves of equal amplitude (symmetric case) travelling in opposite directions. The asymmetric case corresponding to the collision of two waves of unequal amplitudes is also considered.

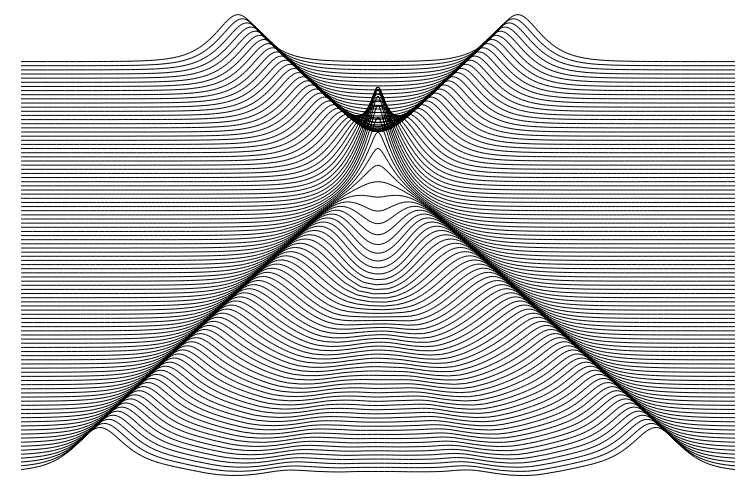

Fig. 2. Free surface profiles at different instants of time for initial amplitude $a / h=0.5$ (from top to bottom).

\section{Numerical simulations and discussion}

3.1 Head-on collision of two solitons of equal amplitude or reflection of high-amplitude solitary wave

\subsubsection{Run-up}

During the wave collision (the runup) the amplitude reaches a value larger than the sum of the amplitudes of the incident solitary waves. After the collision the waves separate into two solitary waves. The collision leaves imprints on the colliding waves with phase shifts and reduced amplitude and shedding a dispersive tail. The temporal evolution of the profile of the free surface corresponding to the head-on collision of solitary waves of amplitude $a / h=0.50$ is given in Fig. 2 . We can observe the dispersive tail when the waves separate after the collision.

The maximum surface elevation defines the maximum value of the runup. The normalized maximum runup, $R / h$, corresponding to the collision of two solitary waves of equal amplitudes is plotted in Fig. 3 as a function of the normalized amplitude of two incident solitons, $a / h$. Our results are in very good agreement with those of Cooker et al. (1997) and extend previous studies to higher values of $a / h$. Within the framework of the fully nonlinear water wave problem, the latter authors investigated the collision of a solitary wave with a vertical wall by using a Boundary Integral Equation Method. In addition, in Fig. 3 are plotted the curves corresponding to analytical results found in the papers by $\mathrm{Su}$ and Mirie (1980) and Pelinovsky et al. (1999) respectively. The former authors obtained to third-order the following expression

$\frac{R}{h}=2 \frac{a}{h}+\frac{1}{2}\left(\frac{a}{h}\right)^{2}+\frac{3}{4}\left(\frac{a}{h}\right)^{3}$.

In the first-order of approximation they considered two independent moving solitary waves which satisfy the KdV equation. Figure 3 shows that the analytical expression derived 


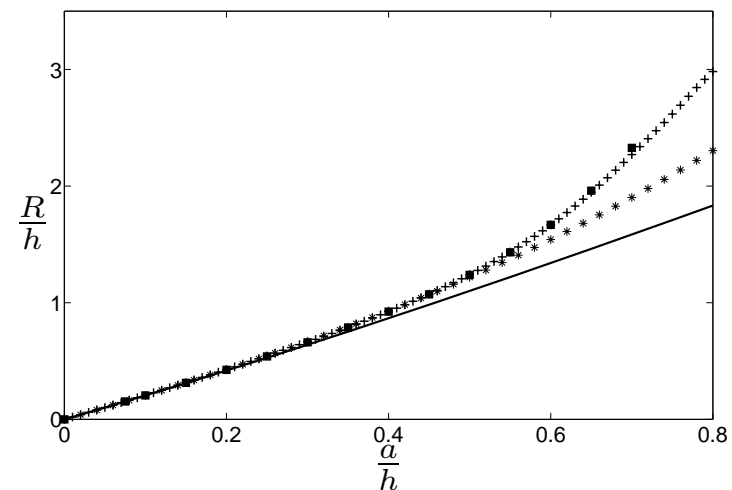

Fig. 3. Normalized maximum runup for a collision of two solitary waves as a function of $a / h,+$ present results, $*$ Su and Mirie (1980), -- Pelinovsky et al. (1999), $\mathbf{c}$ Cooker et al. (1997).

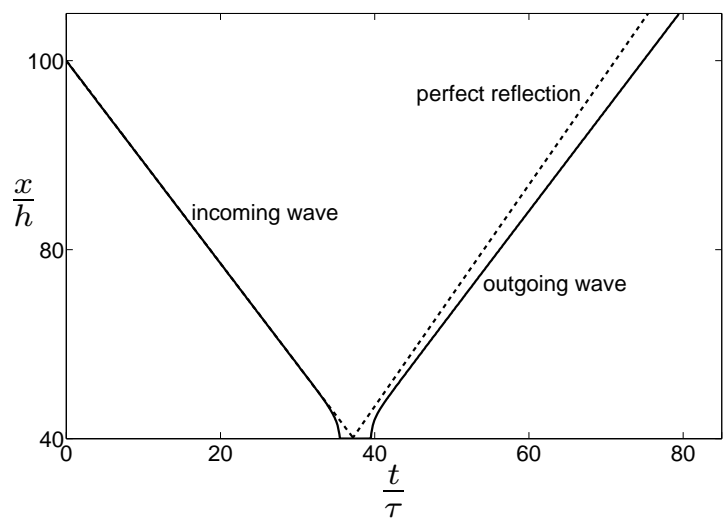

Fig. 4. Schematic diagram of the wave crest trajectory.

by Su and Mirie (1980) gives results which are in excellent agreement with the numerical results up to $a / h=0.50$.

Pelinovsky et al. (1999) used a different approach. They considered the Riemann invariants of the hyperbolic equations of the nonlinear shallow water wave equations to obtain

$\frac{R}{h}=4\left(1+\frac{a}{h}-\sqrt{1+\frac{a}{h}}\right)$.

Let $t_{0}$ be the time of maximum runup at the wall. The time origin is chosen to be the time when the crest of the solitary wave meets the vertical wall within the framework of a perfect reflection (see Fig. 4). Cooker et al. (1997) derived an analytical solution for $t_{0}$ using Su \& Mirie's solution for elevation at second-order of approximation during the runup

$$
\begin{aligned}
\frac{t_{0}}{\tau}= & \frac{1}{2 \sqrt{3}}\left(\left(\frac{a}{h}\right)^{\frac{1}{2}}+\frac{43}{8}\left(\frac{a}{h}\right)^{\frac{3}{2}}\right) \\
& +O\left(\left(\frac{a}{h}\right)^{2}\right) .
\end{aligned}
$$

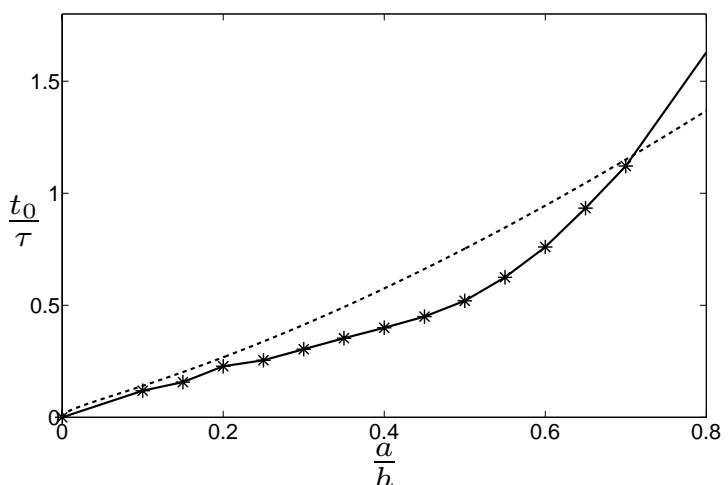

Fig. 5. Normalized time at maximum runup as a function of normalized incident wave amplitude $a / h$; - present results, $*$ Cooker et al. (1997), - - - Su and Mirie (1980).

We calculated this maximum runup time numerically and compared our results with the analytical solution and with the numerical results of Cooker et al. (1997) (Fig. 5). We can observe that the analytical solution is close to the fully nonlinear solution for small values of the amplitude $(a / h \leq 0.20)$ but more amazing for a value of the normalized amplitude near 0.70 .

\subsubsection{Residence time}

The trajectories of the incident wave crest (incoming wave) and reflected wave crest (outgoing wave) is schematically described in the plan $(x / h, t / \tau)$ where $\tau=\sqrt{g / h}$ (see Fig. 4). Note the increase of the phase velocity in the vicinity of the vertical wall. Due to the nonlinear interaction between the reflected wave and the wall (or nonlinear interaction between the right- and left-going solitary waves), the phase velocity of the reflected (or outgoing) wave is less than that of the incoming wave. The wave crest lingers at the wall during reflection for a period of time denoted $t_{r}$. Let $t_{a}$ and $t_{d}$ be the attachment time and detachment time respectively at which the incident wave crest reaches and leaves the vertical wall. Hence, the wall residence time is $t_{r}=t_{d}-t_{a}$. This period of time is an alternative measure of the effects of the wall on the wave or the effect of the nonlinear interaction between the two solitary waves on their phase. Temperville (1979) was the first to report the leading-order asymptotic formula for the wall residence time. His result was independently confirmed by Power and Chwang (1984). The wall residence time may be written in the following form

$$
\begin{aligned}
\frac{t_{r}}{\tau}= & \frac{2}{\sqrt{3}} \ln \left(\frac{\sqrt{3}+1}{\sqrt{3}-1}\right)\left(\frac{a}{h}\right)^{-\frac{1}{2}} \\
& +O\left(\left(\frac{a}{h}\right)^{\frac{1}{2}}\right) .
\end{aligned}
$$




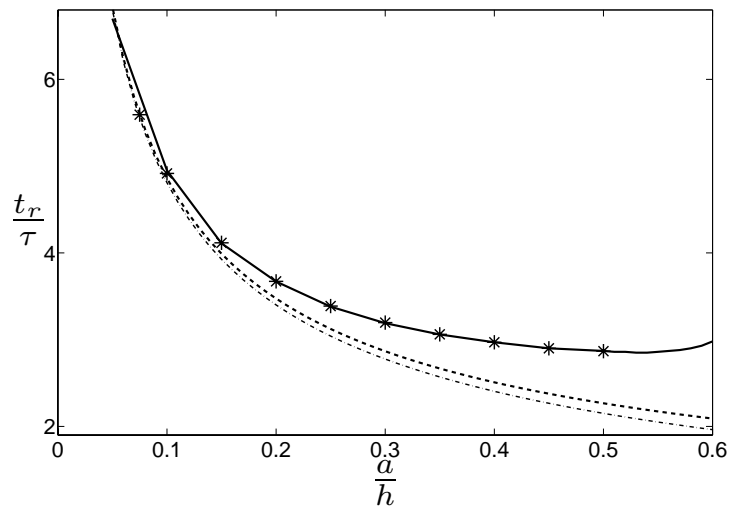

Fig. 6. Normalized wall residence time $t_{r}$ as a function of normalized amplitude $a / h, \ldots$ present results, * Cooker et al. (1997), - - Power and Chwang (1984), - - - Su and Mirie (1980).

From the results of Su and Mirie (1980) we can obtain the residence time to third-order :

$$
\begin{aligned}
\frac{t_{r}}{\tau}= & \frac{2}{\sqrt{3}} \ln \left(\frac{\sqrt{3}+1}{\sqrt{3}-1}\right)\left(\frac{a}{h}\right)^{-\frac{1}{2}} \\
& +\frac{1}{8} \ln \left(\frac{\sqrt{3}+1}{\sqrt{3}-1}\right)\left(\frac{a}{h}\right)^{\frac{1}{2}} .
\end{aligned}
$$

The comparison of our results with previous analytical and numerical works are given in Fig. 6. We have extended beyond $a / h=0.50$ the numerical results obtained by Cooker et al. (1997). For high values of the normalized amplitude $a / h$, the residence time at the wall becomes independent of the amplitude of the incoming wave. Due to the occurrence of a residual jet (see Fig. 16, top) observed for $a / h$ greater than approximately 0.60 , it is not easy to define and calculate $t_{r}$. The weak increase of the residence time in the vicinity of $a / h=0.60$ corresponds to the beginning of the formation of the residual jet.

\subsubsection{Phase shift}

The two solitary waves suffer from a phase shift during the collision process. Similarly, a solitary wave undergoes a phase shift due to the reflection by a vertical wall. Conventionally, the phase shift is the displacement $\Delta x$, the difference in location between the wave crest (solid line in Fig. 4) and the crest of a wave which is supposed to be perfectly reflected (dashed line in Fig. 4) with no change in phase velocity. The phase shift is computed at an instant of time much larger than $t_{d}$ to consider a wave travelling unaffected by the presence of the wall.

Oikawa and Yajima (1973) explicitly computed the spatial phase shift $\Delta x$ incurred after reflection from the wall, namely:

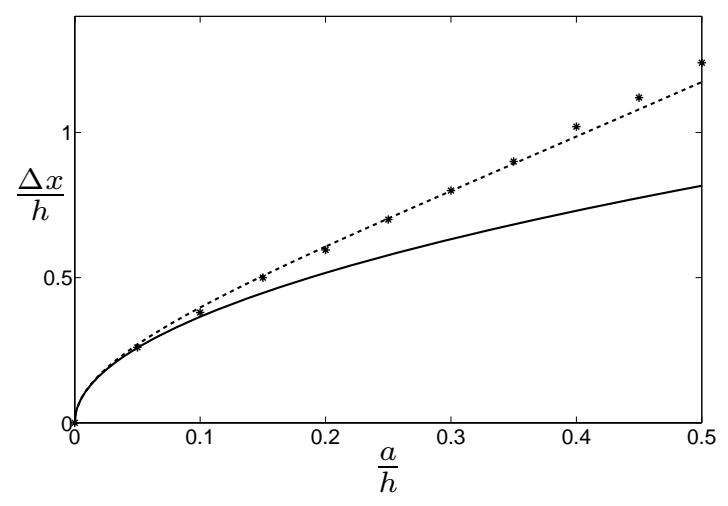

Fig. 7. Normalized phase shift as a function of $a / h$, $*$ Present results, - - - Su and Mirie (1980), — Oikawa and Yajima (1973).

$\frac{\Delta x}{h}=\sqrt{\frac{1}{3} \frac{a}{h}}$.

The improved formulae for the phase shift found by $\mathrm{Su}$ and Mirie (1980) is :

$\frac{\Delta x}{h}=\sqrt{\frac{1}{3} \frac{a}{h}}\left(1+\frac{7}{8} \frac{a}{h}\right)$.

We compare our numerical results with these analytical formulations. The comparison is given in Fig. 7. For $a / h \leq 0.50$, we can observe that the analytical expression derived by $\mathrm{Su}$ and Mirie (1980) is in excellent agreement with the numerical result.

\subsubsection{Accelerations and residual jet formation}

When the amplitude of the two incident solitons is increased above a threshold value a new phenomenon occurs: the formation of a residual jet. The occurrence of this jet is shown in Fig. 8 during the rundown for incident solitary wave amplitude $a / h=0.70$. At $t / \tau=18$ where $\tau=\sqrt{g / h}$, the waves start to separate and leave a residual jet. This jet does not occur in the case corresponding to Fig. 2. From our numerical simulations we found that the residual jet occurs above a threshold value of the normalized amplitude $(a / h)_{c}=0.60$. Figure 10 shows the ephemeral occurrence of a tiny residual jet for incident wave amplitude $a / h=0.60$. For $a / h>0.60$, we have a residual jet formation whereas it is not the case for smaller values.

Figure 9 shows an enlargement of the jet at $t / \tau=18$ corresponding to Fig. 8. The free surface presents undulations which have occurred at the maximum of runup for values of the amplitude of the incident solitons larger than the threshold value, $(a / h)_{c}$, defined previously. Maxworthy (1976) observed experimentally at large initial amplitudes that the vertical accelerations tend to create a jet-like flow at the 


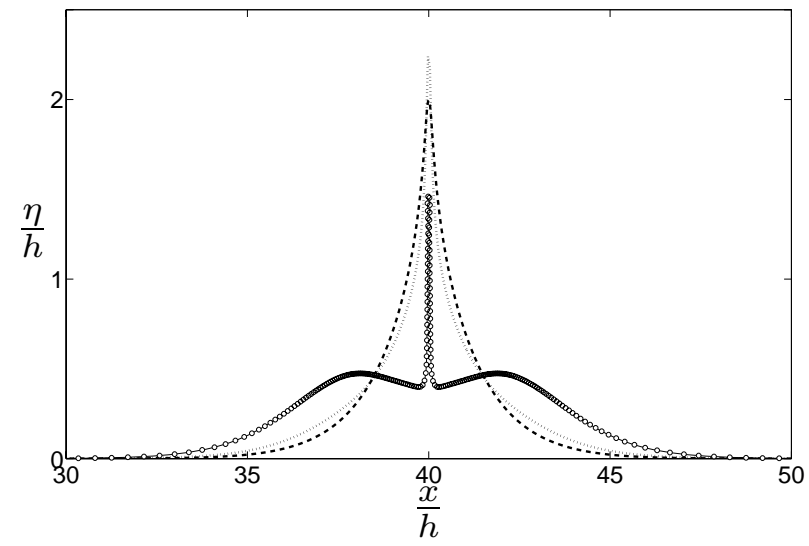

Fig. 8. Rundown for initial amplitude $a / h=0.70$ : Residual jet formation; $\cdots t / \tau=16 ;---t / \tau=17$; $\circ t / \tau=18$.

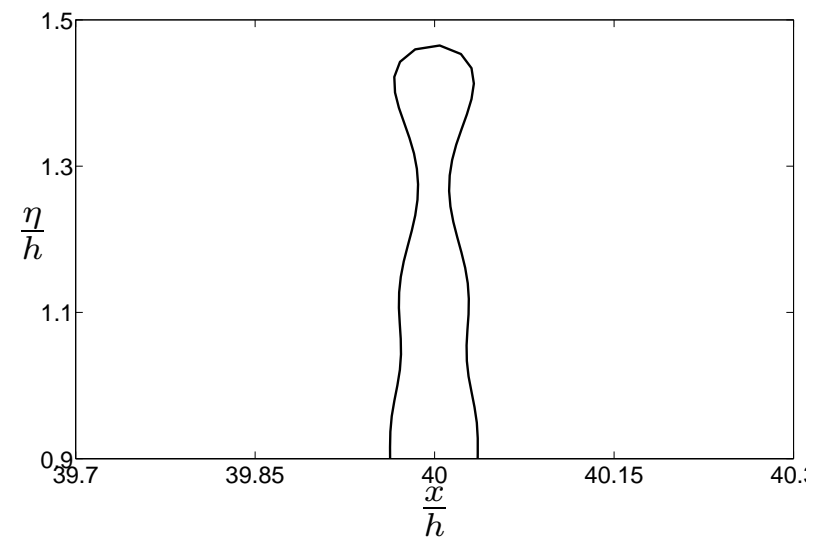

Fig. 9. Residual jet at the free surface for initial amplitude $a / h=0.70$ at $t / \tau=18$.

wave crest which breaks down into individual drops (Fig. 7 in his paper). We suppose we are describing the same phenomenon. Figure 9 shows the initial formation of drops. The drop formation depends on the characteristic time scale of the instability which develops at the free surface of the residual jet. Does interface reconnection occur before the disappearance of the residual falling jet? To answer this question, the breakup of the jet may be studied using VOF methods (see for instance the paper by Chen et al., 1999). The present numerical Boundary Integral Equation Method cannot capture interface reconnection. However, the first step is to consider, within the framework of potential water waves, the stability analysis of this unsteady and non-periodic flow which is at the top of our agenda for further research. In Figs. 11-16 are plotted the profiles of the surface elevation, vertical and horizontal components of the particle acceleration at the free surface during the runup and rundown at several instants of time for different values of the initial amplitude of the solitary waves. For $a / h=0.50$ or 0.70 the head-on collision oc-

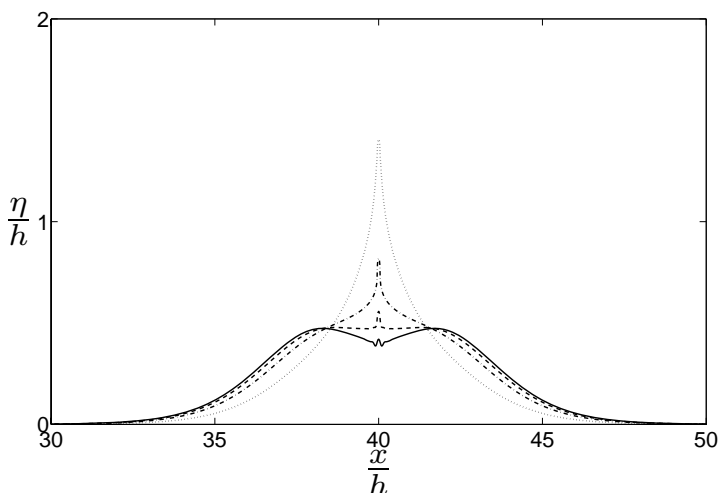

Fig. 10. Rundown for initial amplitude $a / h=0.60$ : Residual jet ; $\cdots t / \tau=17.5 ;-.-t / \tau=18.1 ;---t / \tau=18.3 ;-t / \tau=18.4$.

curs at $x / h=40$ (middle of the tank) whereas for $a / h=0.20$ we have used a longer wave tank. Hence the collision takes place at $x / h=70$ (middle of the tank).

The maximum of the absolute value of the vertical acceleration $a_{z}$ is obtained at the crest of the free surface at the end of the runup or the beginning of the rundown. This value increases with the amplitude $a / h$ up to a limit, $g$, the acceleration due to gravity, as it is shown in Fig. 17.

For initial amplitudes less than $a / h=0.60$, the intensity of the vertical acceleration is always less than gravity whereas for larger amplitudes the maximum of the ratio $a_{z} / g$ saturates to the value -1 . For $a / h$ larger than 0.60 it is observed during the rundown that accelerations at the crest and in its vicinity remain equal to $-g$ (see Fig. 16, bottom).

During the rundown, when the waves separate after the collision a new phenomenon occurs: the formation of a residual jet. This is shown in Fig. 16 (top) for $a / h=0.70$. The residual jet formation does not occur for collisions corresponding to $a / h=0.20$ or $a / h=0.50$. We found that the residual jet formation starts when the normalized amplitude of two incident solitons is larger than 0.60 . The formation of the residual jet occurs when the crest acceleration is $-g$. This means that the jet is in freefall. We may expect that the residual jet is due to the Rayleigh-Taylor instability. For more details on this mechanism see the paper by Taylor (1950). As mentioned previously, the mechanism of generation of this jet is an open question which will be more deeply investigated in a future research.

During the formation of the residual jet the crest curvature becomes important. Hence we may wonder what is the effect of surface tension on the formation and evolution of the residual jet. We consider the surface tension effect on the dynamics of the collision. The relative strength of gravity effects to capillary effects is measured through the Bond number $B o=\rho \mathrm{gh}^{2} / \sigma$. For the present case, the water density is $\rho=10^{3} \mathrm{~kg} . \mathrm{m}^{-3}$, the acceleration due to gravity is $g=9.80 \mathrm{~m} \cdot \mathrm{s}^{-2}$, the water depth is $h=1 \mathrm{~m}$ and the surface 

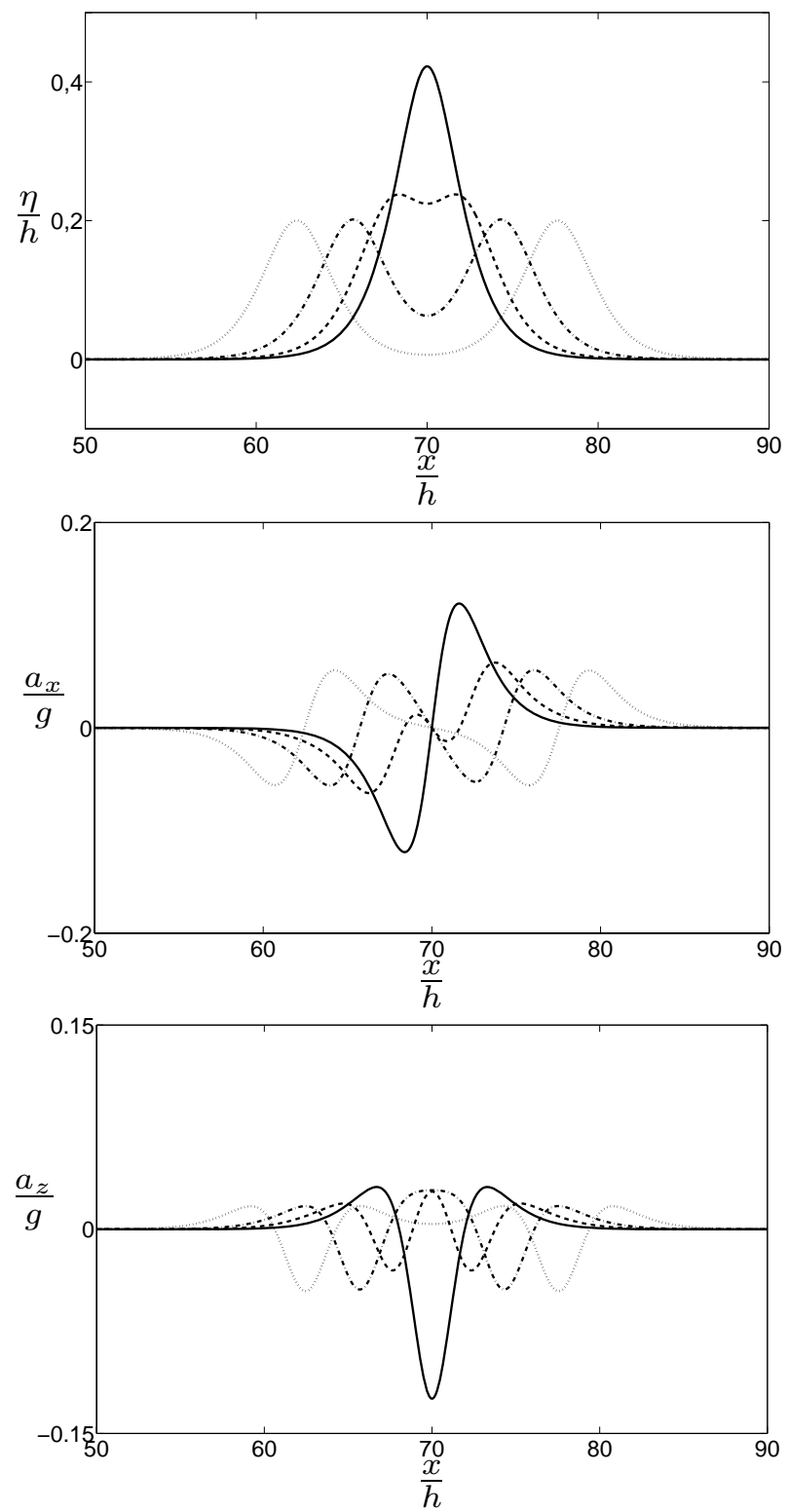

Fig. 11. Runup for soliton amplitude $a / h=0.2$.

Free surface elevation and horizontal and vertical accelerations (from top to bottom) at several instants of time. $\cdots t / \tau=25$, $-\cdot-t / \tau=28,---t / \tau=30,-t / \tau=32$.

tension coefficient is $\sigma=74 \times 10^{3} \mathrm{~N} \cdot \mathrm{m}^{-1}$. The Bond number in our dimensionless system is $B o=1.32 \times 10^{6}$. We can conclude that gravity forces are globally dominant. Nevertheless, surface tension effect cannot be neglected locally where the free surface curvature is important. Capillarity starts to play a role on the dynamics of the residual jet during the rundown as it is shown in Fig. 18. Surface tension which has a stabilizing effect does not avoid the formation of the residual jet. This feature lead us to believe that the occurrence of the residual jet is not an numerical artefact of the present method.
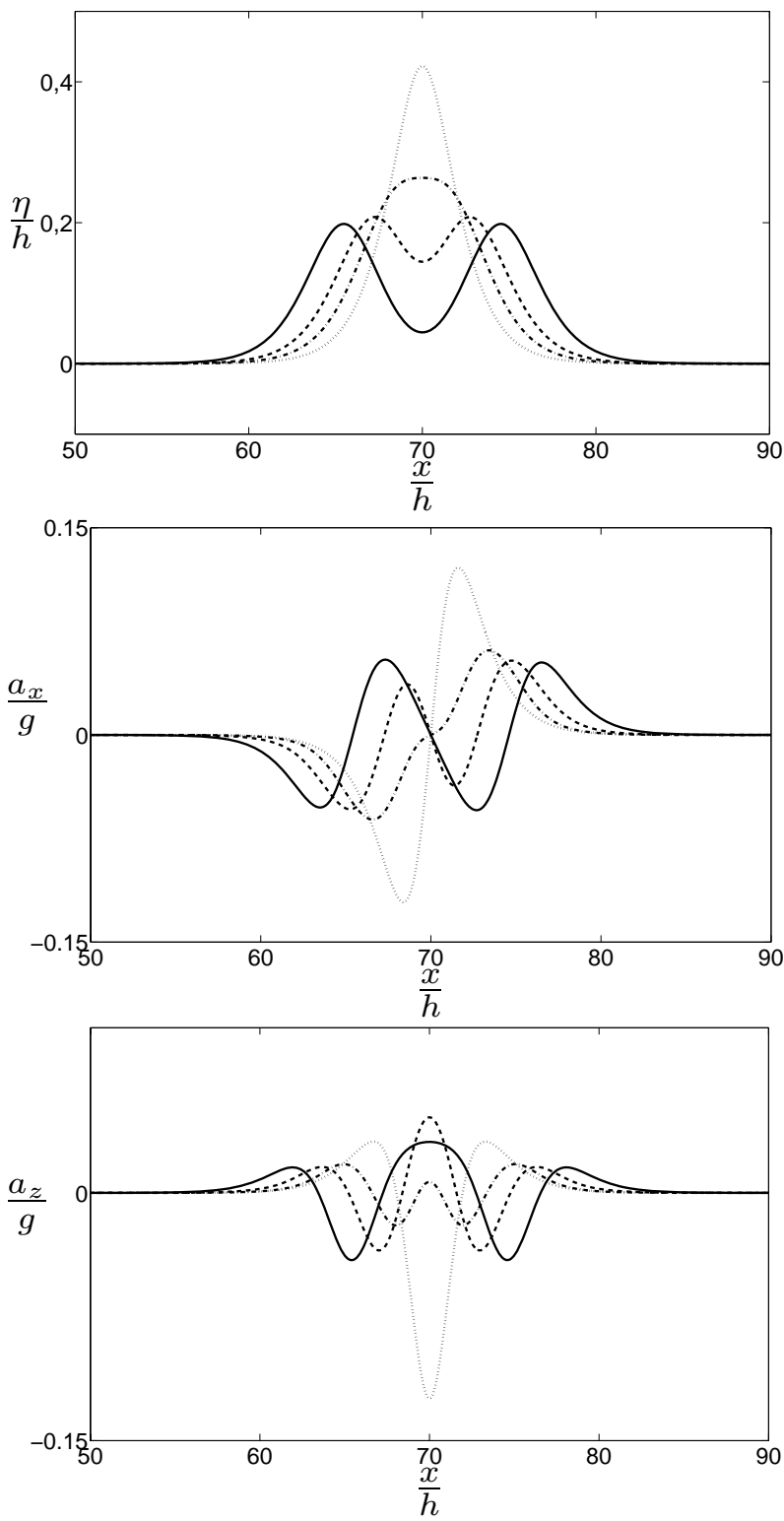

Fig. 12. Rundown for soliton amplitude $a / h=0.2$.

Free surface elevation and horizontal and vertical accelerations (from top to bottom) at several instants of time. $\cdots t / \tau=32$, $-\cdot-t / \tau=34,---t / \tau=35,-t / \tau=36.5$.

\subsubsection{Instantaneous wall force}

The head-on collision of two equal solitary waves is equivalent to the reflexion of one solitary wave by a vertical wall. Following Cooker et al. (1997) we have computed the instantaneous wall force up to $a / h=0.8$. The wall is assumed to be located in the middle of the numerical tank where the collision of the two solitary waves occurs. Figure 19 shows the instantaneous wall force $F_{w}$ during the runup as a function of time relative to $t_{0}$. To compute the wall force, the pressure 

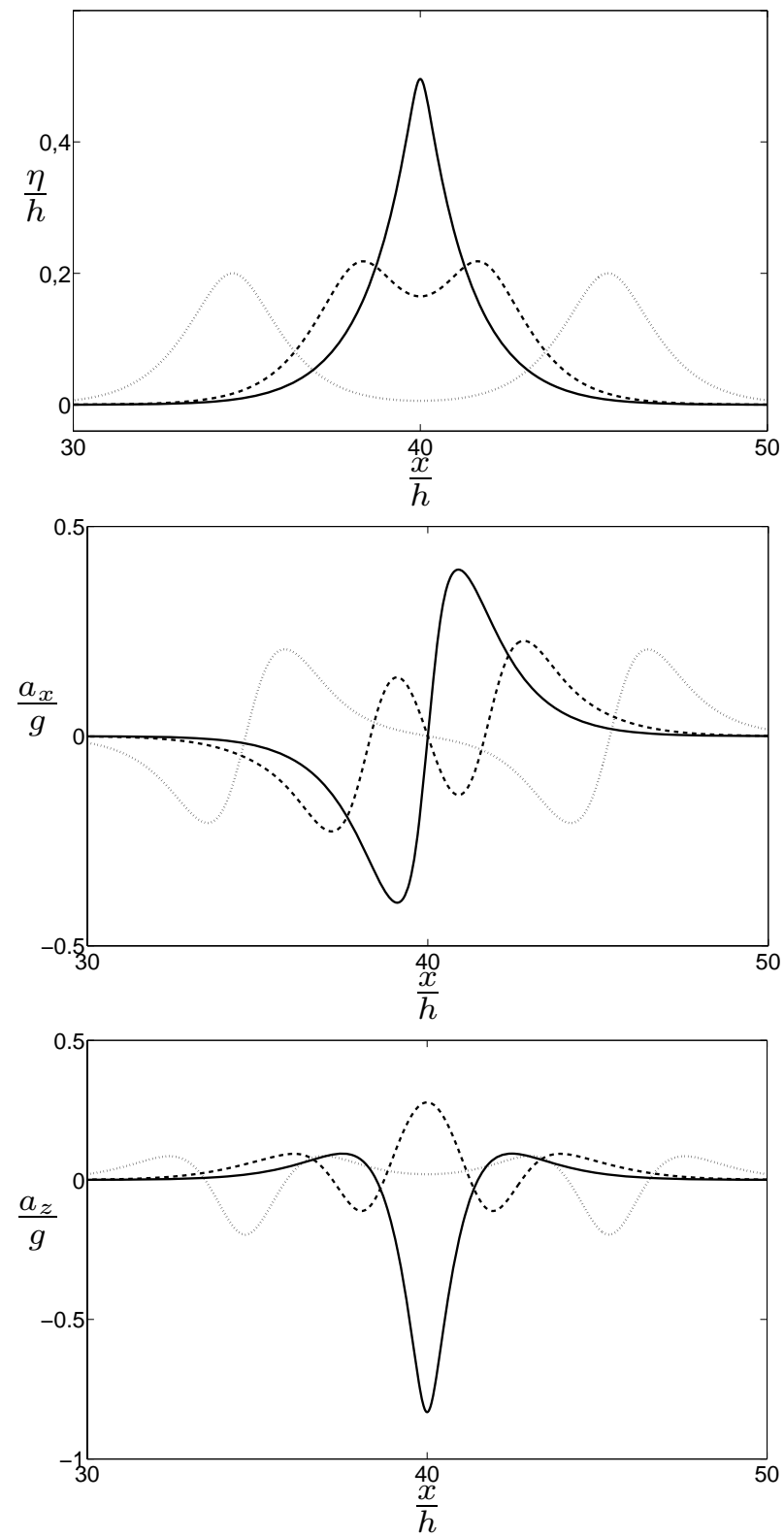

Fig. 13. Runup for soliton amplitude $a / h=0.5$.

Free surface elevation and horizontal and vertical accelerations (from top to bottom) at several instants of time. $\cdots t / \tau=12$, $--t / \tau=15,-t / \tau=17$.

$P(z)$ along the vertical wall is determined by using Bernoulli equation :

$\frac{P(z)}{\rho}=-\frac{\partial \Phi}{\partial t}-\frac{1}{2}(\nabla \Phi)^{2}-g z$.
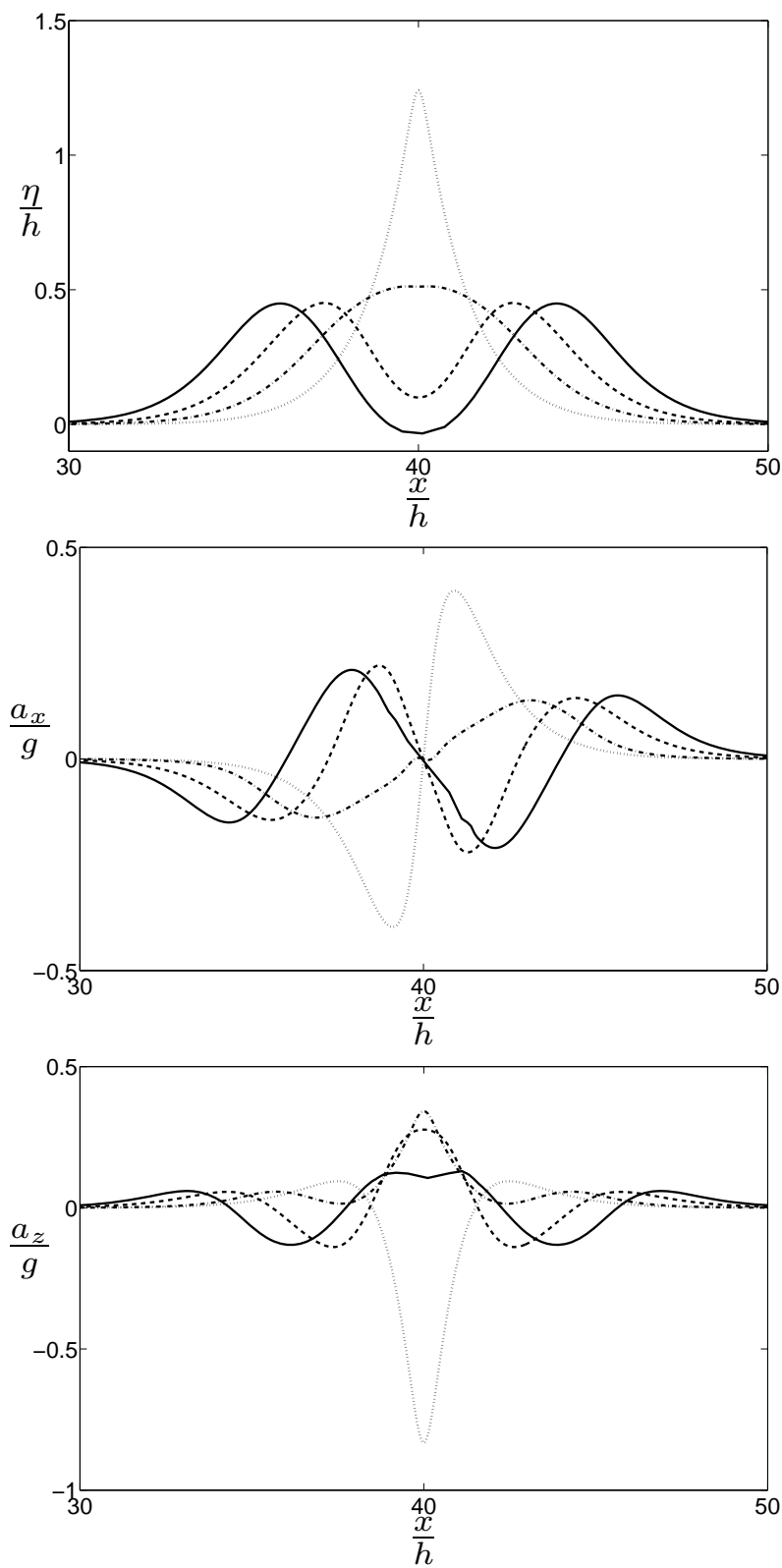

Fig. 14. Rundown for soliton amplitude $a / h=0.5$.

Free surface elevation and horizontal and vertical accelerations (from top to bottom) at several instants of time. $\cdots t / \tau=17$, $-\cdot-t / \tau=18.5,---t / \tau=19.5,-t / \tau=20.5$.

Hence, the wall force is given by :

$F_{w}=\int_{-h}^{\eta} P(z) d z$

Our results are in very good agreement with those of Cooker et al. (1997) as shown in Fig. 19. For $a / h<0.3$ the maximum force occurs at maximum runup, the pressure in 

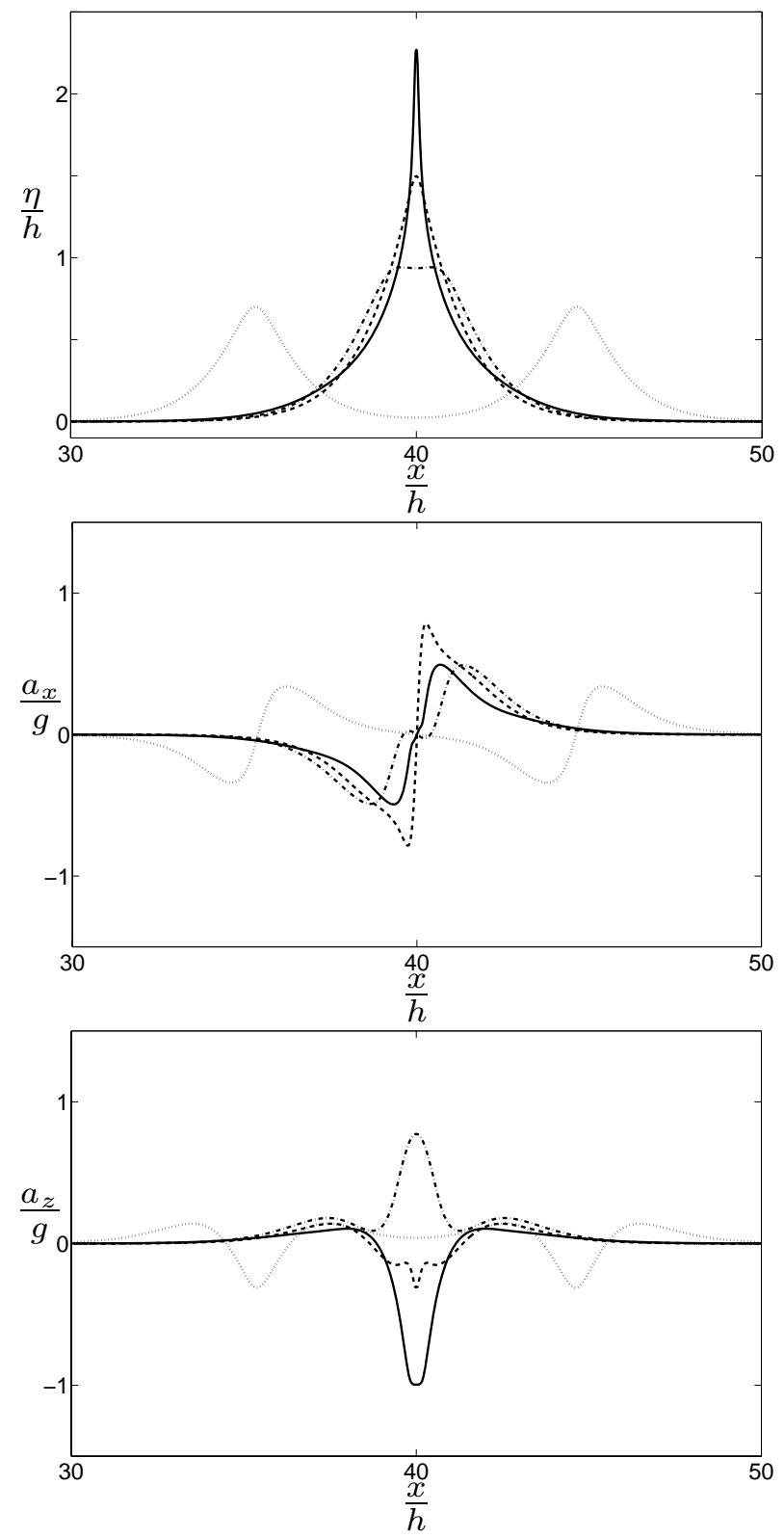

Fig. 15. Runup for soliton amplitude $a / h=0.7$.

Free surface elevation and horizontal and vertical accelerations (from top to bottom) at several instants of time. $\cdots t / \tau=12$, $-\cdot-t / \tau=15,---t / \tau=15.5,-t / \tau=16.35$.

the fluid is like in hydrostatic case. For $a / h>0.4$ the vertical acceleration of the free surface is significant during the runup. As discussed by Cooker et al. (1997) the maximum force is obtained before maximum runup because the upsurging wave forms a narrow jet. For large $a / h$ values, a second maximum force occurs because there is another increase in the fluid pressure. For their case $a / h=0.7$ Cooker et al. (1997) were not sure whether there is also a second peak past $\left(t-t_{0}\right) / \tau=0$. Our computations confirm that there is indeed
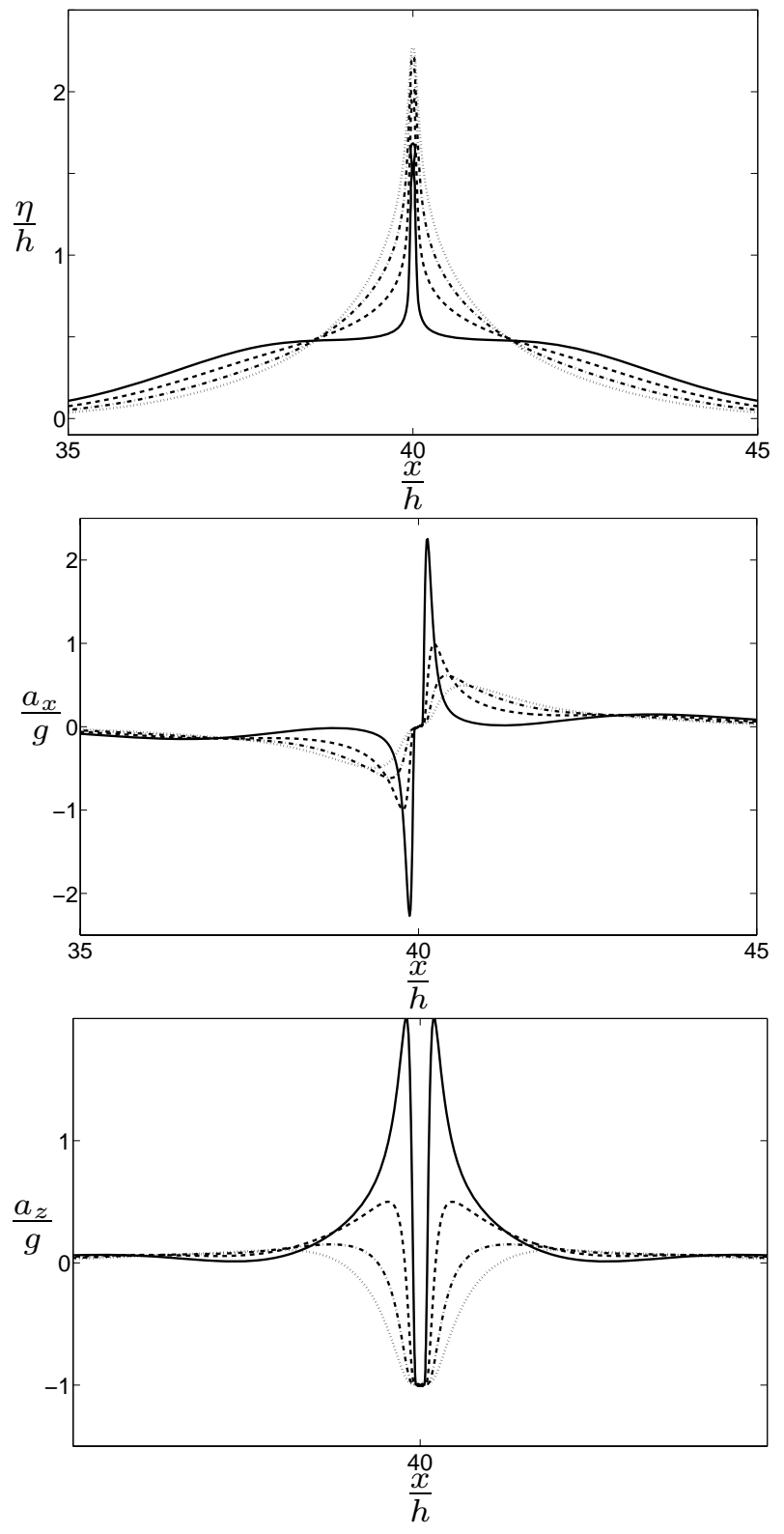

Fig. 16. Rundown for soliton amplitude $a / h=0.7$.

Free surface elevation and horizontal and vertical accelerations (from top to bottom) at several instants of time. $\cdots \cdot t / \tau=16.35$, $-\cdot-t / \tau=16.55,---t / \tau=16.75,-t / \tau=16.95$.

a second peak for the case $a / h=0.7$. We have extended the calculation of the wall force to $a / h=0.8$ and obtained a similar behaviour. We plot in Fig. 20 the maximum instantaneous force $\left(F_{w}\right)_{m}$ as function of $a / h$. Our results are in agreement with those of Cooker et al. (1997). 


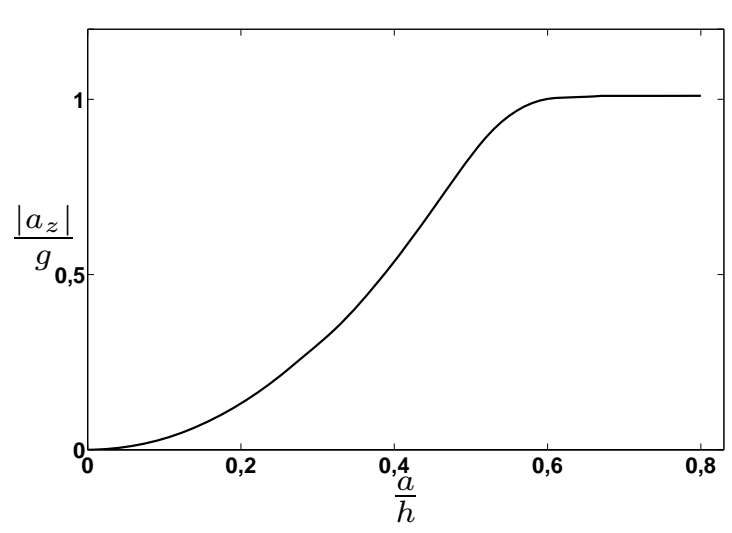

Fig. 17. Evolution of the maximum of vertical acceleration as a function of $a / h$.

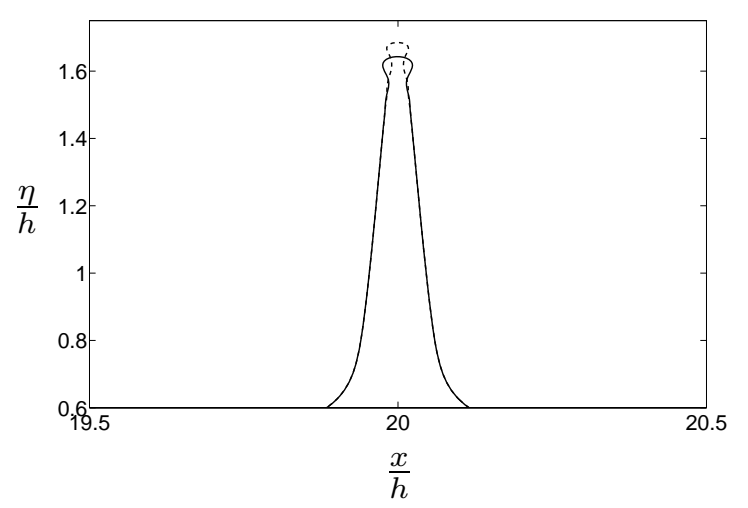

Fig. 18. Residual falling jet for initial amplitude $a / h=0.69$ with and without surface tension: jet with surface tension (solid line), jet without surface tension (dashed line).

\subsection{Head-on collision of two unequal amplitude solitary} waves

In this section we consider the collision of two solitary waves with different amplitudes $a_{r} / h$ and $a_{l} / h$. Indices $r$ and $l$ denote waves propagating from left to right and from right to left, respectively.

Figures 21 and 22 show free surface profiles at several instant of time for two numerical experiments corresponding to $\left(a_{r} / h, a_{l} / h\right)=(0.40,0.10)$ and $\left(a_{r} / h, a_{l} / h\right)=(0.70,0.80)$, respectively. During the maximum of runup the wave presents an asymmetric profile. This asymmetry increases with the wave amplitude of the two solitons. For large values of the amplitude, an oblique residual jet develops during the rundown as shown in Fig. 22. Similarly to the symmetric case the solitons suffer from phase shift. A comparison between the analytical results obtained by Su and Mirie (1980) and presents results is given in Table 1. In the table are given for several value on the two initial solitary waves the max-

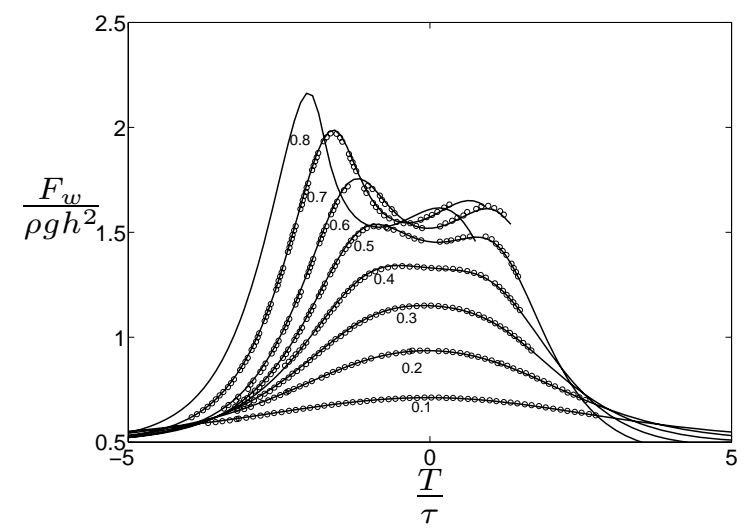

Fig. 19. Instantaneous wall force as function of time relative to $t_{0}\left(T=t-t_{0}\right)$ for selected values of $a / h . \circ$ Cooker et al. (1997); present results.

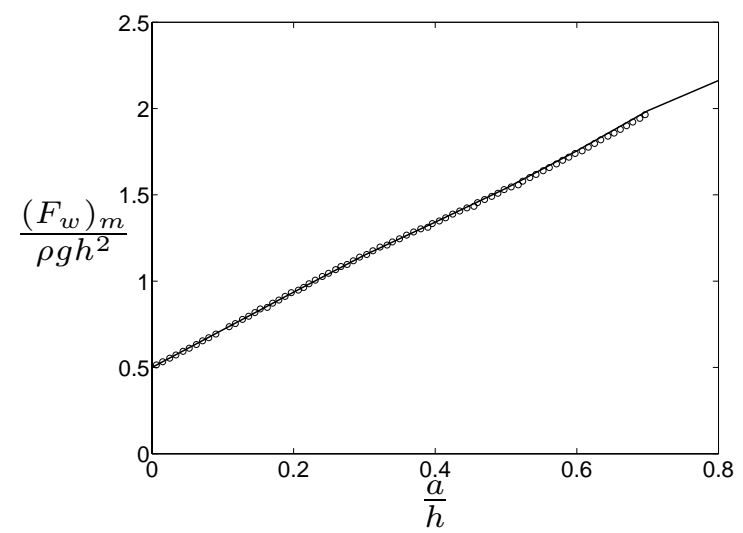

Fig. 20. Maximum instantaneous force plotted as function of $a / h$.० Cooker et al. (1997); — present results.

imum runup, $R / h$, phase shifts due to the collision of the two solitons, $\Delta x_{r} / h$ and $\Delta x_{l} / h$ computed numerically and the corresponding analytical results derived by $\mathrm{Su}$ and Mirie (1980), $R_{S M} / h, \Delta x_{r S M} / h$ and $\Delta x_{l S M} / h$ :

$\frac{R_{S M}}{h}=\frac{a_{r}}{h}+\frac{a_{l}}{h}+\frac{1}{2} \frac{a_{r} a_{l}}{h^{2}}+\frac{3}{8} \frac{a_{r} a_{l}}{h^{2}}\left(\frac{a_{r}}{h}+\frac{a_{l}}{h}\right)$,

$\frac{\Delta x_{r S M}}{h}=\left(\frac{1}{3} \frac{a_{l}}{h}\right)^{\frac{1}{2}}\left(1+\frac{1}{8} \frac{a_{l}}{h}+\frac{3}{4} \frac{a_{r}}{h}\right)$,

$\frac{\Delta x_{l S M}}{h}=-\left(\frac{1}{3} \frac{a_{r}}{h}\right)^{\frac{1}{2}}\left(1+\frac{1}{8} \frac{a_{r}}{h}+\frac{3}{4} \frac{a_{l}}{h}\right)$.

For the runup, we found an excellent agreement between our numerical results and those obtained from Su and Mirie's analytical expression whereas for phase shifts the deviation is larger. 
Table 1. Comparison between present and Su and Mirie (1980) results for the maximum runup and phase shifts for several values of the amplitudes of the two solitary waves.

\begin{tabular}{cccccccc}
\hline$a_{r} / h$ & $a_{l} / h$ & $R / h$ & $R_{S M} / h$ & $\Delta x_{r} / h$ & $\Delta x_{r S M} / h$ & $\Delta x_{l} / h$ & $\left|\Delta x_{l S M}\right| / h$ \\
\hline 0.40 & 0.10 & 0.5239 & 0.5275 & 0.4401 & 0.4108 & 0.2152 & 0.2396 \\
0.40 & 0.30 & 0.7867 & 0.7915 & 0.5000 & 0.4656 & 0.4353 & 0.4230 \\
0.50 & 0.25 & 0.8422 & 0.8477 & 0.5627 & 0.5103 & 0.3894 & 0.4059 \\
0.60 & 0.20 & 0.8903 & 0.8960 & 0.6100 & 0.5478 & 0.3469 & 0.3808 \\
0.80 & 0.70 & 2.6013 & 2.0950 & - & 0.8391 & - & 0.8151 \\
\hline
\end{tabular}
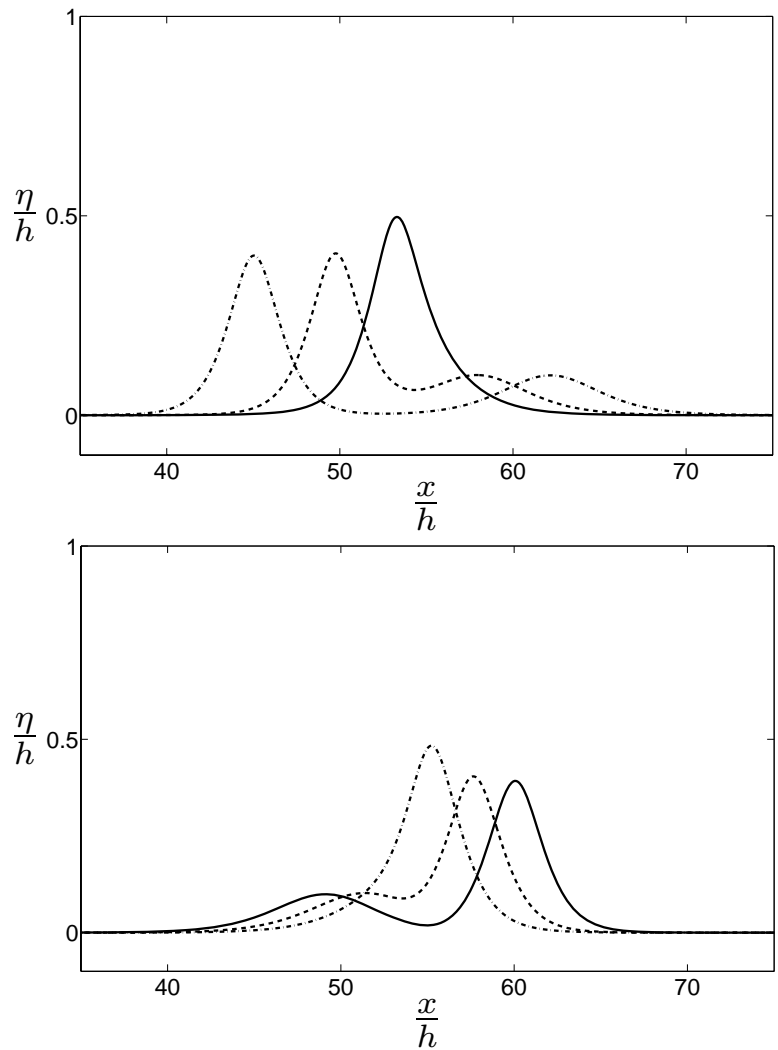

Fig. 21. Free surface elevation corresponding to counter propagating solitary waves with amplitudes $a_{r} / h=0.40$ and $a_{l} / h=0.10$ at several instants of time. (Top) $-\cdot-t / \tau=17,---t / \tau=21$, $-t / \tau=24$. (Bottom) $-\cdot-t / \tau=26,---t / \tau=28,-t / \tau=30$.

\section{Conclusions}

The present paper has extended the previous results of the literature on the maximum runup, wall residence time and phase shift, to very high values of the amplitude of two identical counter propagating solitons. We used the classical Boundary Integral Equation Method considering when necessary surface tension effects. We observe the same results with and without surface tension. Moreover, the particle acceleration at the free surface has been computed. A new phenomenon has been discovered corresponding to
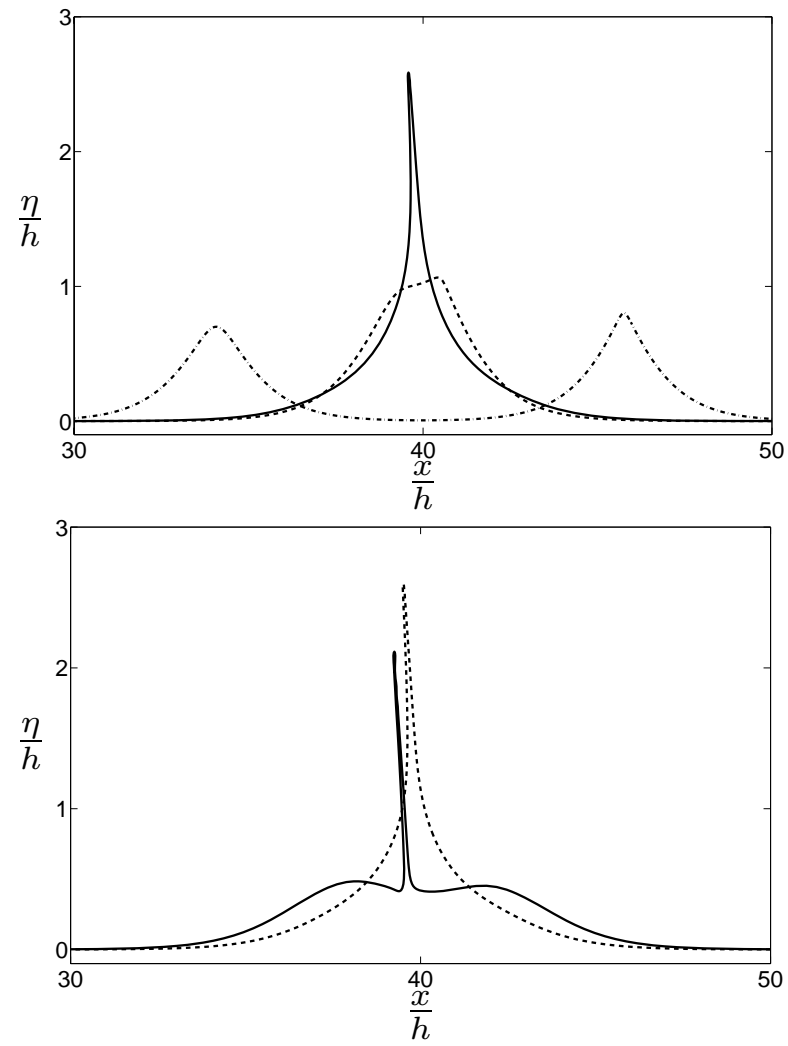

Fig. 22. Free surface elevation corresponding to counter propagating solitary waves with amplitudes $a_{r} / h=0.70$ and $a_{l} / h=0.80$ at several instants of time. (Top) $-\cdot-t / \tau=11,---t / \tau=15$, $-t / \tau=16.65$. (Bottom) $---t / \tau=16.95,-t / \tau=17.95$.

the occurrence of a thin residual jet when the normalized amplitude of the solitons is larger than 0.60 . We believe that the jet observed experimentally by Maxworthy (1976) is the same phenomenon. The mechanism of generation of this jet is still an open problem. We also computed the instantaneous force on the symmetric axis of the collision. The asymmetric case corresponding to two counter propagating solitary waves of different amplitudes has been investigated, too. Numerical computations of the maximum runup and phase shifts have been compared with analytical results provided by $\mathrm{Su}$ and Mirie (1980). Like the symmetric case, a thin 
residual jet occurs for high values of the amplitude. Note that the residual jet is now oblique. Additional stability analysis and experiments are needed to confirm the present findings.

Edited by: I. Didenkulova

Reviewed by: two anonymous referees

\section{References}

Bona, J. L. and Chen, M.: A Boussinesq system for two-way propagation of nonlinear dispersive waves, Physica D., 116, 191-224, 1998.

Byatt-Smith, J. G. B.: An integral equation for unsteady surface waves and a comment on the Boussinesq equation., J. Fluid Mech., 49, 625-633, 1971.

Byatt-Smith, J. G. B.: The reflection of a solitary wave by a vertical wall, J. Fluid. Mech., 197, 503-521, 1988.

Chan, R. K. C. and Street, R. L.: A computer study of finiteamplitude water waves., J. Computat. Phys., 6, 68-94, 1970.

Chen, G., Kharif, C., Zaleski, S., and Li, J.: Two-dimensional Navier-Stokes simulation of breaking waves., Phys. Fluids, 11, 121-133, 1999.

Cooker, M. J., Weidman, P. D., and Bale, D. S.: Reflection of a high-amplitude solitary wave at a vertical wall, J. Fluid Mech., 342, 141-158, 1997.

Craig, W., Guyenne, P., Hammack, J., Henderson, D., and Sulem, C.: Solitary wave interactions., Phys. Fluids, 18, 1-25, 2006.

Fenton, J. D. and Rienecker, M. M.: A Fourier method for solving nonlinear water-wave problems : application to solitary-wave interactions., J. Fluid Mech., 118, 411-443, 1982.
Maxworthy, T.: Experiments on collisions between solitary waves., J. Fluid Mech., 76, 177-185, 1976.

Mirie, R. M. and Su, C. H.: Collisions between two solitary waves. Part 2. A numerical study., J. Fluid. Mech., 115, 475-492, 1982.

Oikawa, M. and Yajima, N.: Interactions of solitary waves - a perturbation approach to nonlinear systems., J. Phys. Soc. Japan, 34, 1093-1099, 1973.

Pelinovsky, E., Troshina, E., Golinko, V., Osipenko, N., and Petrukhin, N.: Runup of tsunami waves on a vertical wall in a basin of complex topography., Phys. Chem. Earth (B), 24, 431436, 1999.

Power, H. and Chwang, A. T.: On reflection of a planar at a vertical wall., Wave Motion, 6, 183-195, 1984.

Su, C. H. and Gardner, C. S.: Korteweg-de Vries equation and generalizations. III. Derivation of the Korteweg-de vries equation and Burgers equation., J. Math. Phys., 10, 536-539, 1969.

$\mathrm{Su}, \mathrm{C}$. H. and Mirie, R. M.: On head-on collisions between two solitary waves., J. Fluid Mech., 98, 509-525, 1980.

Tanaka, M.: The stability of solitary waves., Phys. Fluids, 29, 650655, 1986.

Taylor, S. G.: The instability of liquid surfaces when accelerated in a direction perpendicular to their planes, Proc. Roy. Soc. London, 201, 192-196, 1950.

Temperville, A.: Interaction of solitary waves in shallow water theory., Arch. Mech., 31, 177-184, 1979.

Touboul, J. and Kharif, C.: Two-dimensional direct numerical simulations of the dynamics of rogue waves under wind action (Ed. Q. W. Ma), The world Scientific Publishing Co, 2009.

Touboul, J., Giovanangeli, J. P., Kharif, C., and Pelinovsky, E.: Freak waves under the action of wind: Experiments and simulations, Eur. J. Mech. B/Fluids, 25(5), 662-676, 2006. 